\title{
Taking Control of the Time Bomb in Abdominal Aortic Aneurysm
}

\author{
Hiroki Aoki, MD, PhD
}

$\mathbf{Y}$ ou are watching the climax scene of an old 007 movie. James Bond has discovered a time bomb, on which the evil guy was kind enough to put a precise time indicator that shows only 60 seconds are left before the deadly explosion. Mr Bond is struggling to stop the time bomb, occasionally taking a look at the time indicator. Tick, tick...He made it! The bomb has been disabled and the indicator shows it was just 007 seconds before the explosion.

\section{Article p 332}

Having an abdominal aortic aneurysm (AAA) is like living with a time bomb (Figure). The AAA is silently ticking in the patient until it explodes with little or no warning. The good thing is that AAA does have an indicator, like timer of the time bomb in the 007 movie. The diameter of the AAA is a fairly reliable indicator of whether the aneurysm is at high risk of rupture. With the advancement in imaging technologies, AAA diameter remains the gold standard of the risk assessment. ${ }^{1,2}$ The problem is that it is uncertain how fast the "timer" is ticking. Even though a small diameter is indicative of low rupture risk, it is still uncertain when the next check should be scheduled. It would help clinicians to schedule the next check if there is a second indicator that shows how fast the first indicator, the AAA diameter, is ticking time.

In this issue of the Journal, Nakayama et $\mathrm{al}^{3}$ report that calcification of the AAA as measured by X-ray computed tomography (CT) may just fill the requirement of the second indicator. The authors found that the greater the calcification of the AAA, the slower it expands. The AAA diameter and calcification are independent predictors of the speed of expansion of AAA, and the combination of them is a better predictor than diameter alone. Because X-ray CT is routinely used for the diagnosis and monitoring of AAA, this finding has high clinical value because the extent of calcification may provide an important clue for predicting AAA expansion speed and therefore when the next observation should be scheduled. Such a rationalized scheduling of AAA monitoring would be beneficial from both the patient-specific and sociomedical point of view. For a given AAA patient, the optimal observation schedule would provide the confidence that one can get timely intervention before the catastrophic event of AAA rupture without unnecessary exposure to X-ray from frequent CT observations. The patient-specific scheduling of AAA monitoring would also lead to a better cost-benefit for the AAA

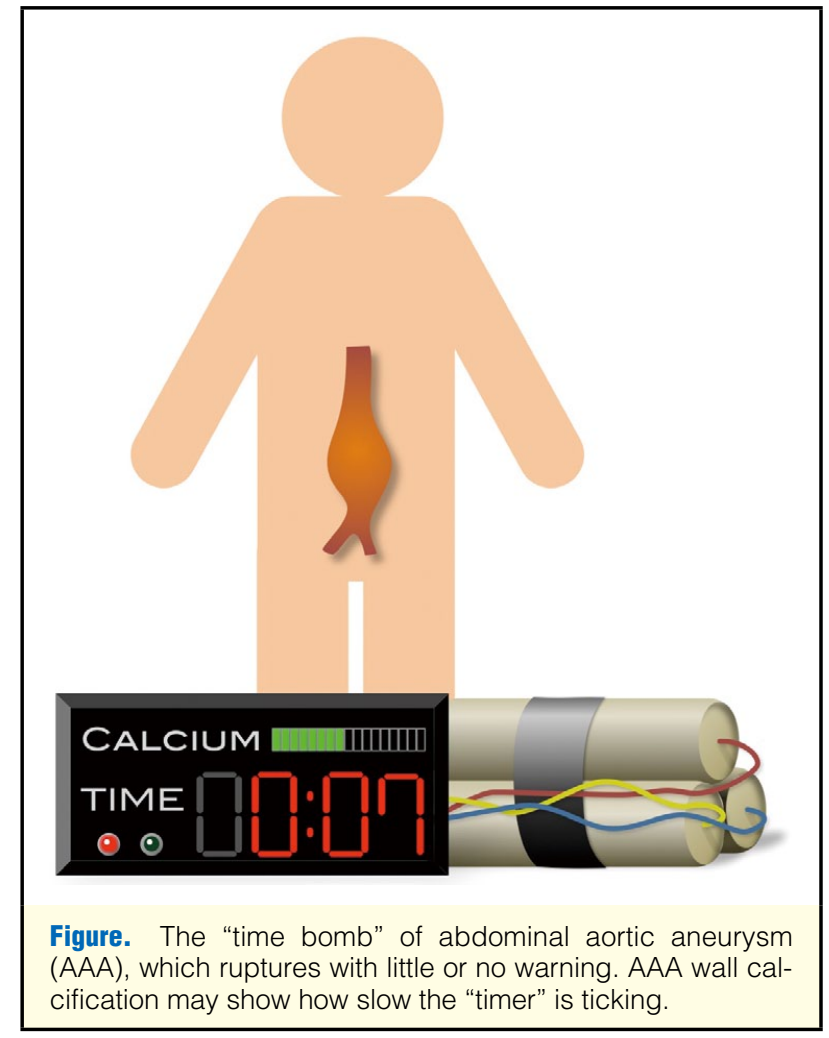

clinical practice. Rationalized scheduling can reduce the cost of unnecessary clinical imaging and of emergency operations in case of AAA rupture.

To realize such scheduling of AAA monitoring, an important issue must be addressed. Does calcification directly affect the rupture risk of AAA, independent of the diameter? If calcification by itself has no or negative effect on the rupture risk of AAA, less frequent observation of a calcified AAA may be justified because the growth of the AAA would be slower. However, it is problematic if calcification positively relates to the rupture risk, because a more calcified AAA may require earlier intervention and more frequent monitoring even though the expansion speed may be slower than a less calcified AAA. As pointed out by the authors, aortic wall calcification by itself

The opinions expressed in this article are not necessarily those of the editors or of the Japanese Circulation Society.

Received December 16, 2015; accepted December 17, 2015; released online January 6, 2016

Cardiovascular Research Institute, Kurume University, Kurume, Japan

Mailing address: Hiroki Aoki, MD, PhD, Cardiovascular Research Institute, Kurume University, 67 Asahimachi, Kurume, Fukuoka

830-0011, Japan. E-mail: haoki@med.kurume-u.ac.jp

ISSN-1346-9843 doi:10.1253/circj.CJ-15-1350

All rights are reserved to the Japanese Circulation Society. For permissions, please e-mail: cj@j-circ.or.jp 
may physically resist AAA expansion by virtue of its hardness. However, the heterogeneity in the physical properties of the aortic wall because of calcification would result in uneven distribution of the wall stress, leading to an increase in the peak stress at the calcification sites. ${ }^{4}$ Indeed, an observational study suggested that calcification of AAA may be a risk factor for rupture. ${ }^{5}$ Studies of this issue would add more value to the current finding. For example, clinical imaging may provide evidence of an association of the calcified and high-stress regions in the wall of the AAA. Molecular imaging such as fluoro-deoxy-glucose positron emission tomography may detect the association of inflammation that promotes wall destruction and rupture ${ }^{6}$ and calcification. It would also help to perform histopathological studies of AAA tissue excised during the surgical repair to see if and how calcification is associated with the inflammation and destruction of AAA walls. Such studies would also advance our understanding of the pathogenesis of AAA.

Another interesting issue is why a more calcified AAA expands less rapidly. AAA has been considered an atherosclerotic disease because the aortic wall destruction resulting from atherosclerotic changes makes the wall weaker, predisposing the aorta to the development of AAA. This assumption has been made mainly because most AAA are associated with severe atherosclerosis, and the risk factors of AAA partly overlap with those of atherosclerosis. However, no formal proof has been made for causality between atherosclerosis and AAA development. It is even more questionable whether atherosclerosis promotes the progression of AAA. Indeed, diabetes mellitus, a strong promoter of atherosclerosis, has been proven a strong negative risk factor for the prevalence and progression of AAA. ${ }^{7}$ Because calcification is indicative of more severe and complicated atherosclerosis, the current study ${ }^{3}$ may provide more evidence that AAA and atherosclerosis are different and possibly even opposite pathological entities.

The mechanism of calcification by itself and how the process is involved in pathogenesis of AAA are of interest. While arterial wall calcification used to be thought as just a consequence of inflammation, it is now evident that the osteogenic differentiation of vascular smooth muscle cells participates in arterial wall calcification. ${ }^{8}$ This suggests that AAA wall calcification may also be a regulated process that possibly offers the opportunities both for the monitoring disease activities and for the therapeutic interventions. Because the changes in AAA wall calcification can be precisely measured as shown in the current study, ${ }^{3}$ this could be a quantitative index to monitor the disease activity. Also, if calcification reflects a particular aspect of the AAA pathogenesis, its change may be utilized to monitor the efficacy of the future non-surgical treatment. It may also be possible to promote or prevent the AAA calcification, depending on whether calcification is the risk of AAA rupture or not.

As our society ages, the prevalence of AAA will dramatically increase, as a recent study demonstrated, ${ }^{9}$ which will have a significant effect on the sociomedical burden in the future. The significance of the current findings may increase upon elucidation of the mechanism and consequence of the AAA wall calcification, as discussed above. Once these issues are addressed, our James Bond, the physicians and the surgeons fighting against AAA, may have a good chance of disabling the bomb in a timely manner by watching a more precise indicator of AAA rupture.

\section{References}

1. JCS Joint Working Group. Guidelines for diagnosis and treatment of aortic aneurysm and aortic dissection (JCS 2011): Digest version. Circ J 2013; 77: 789-828.

2. Buijs RV, Willems TP, Tio RA, Boersma HH, Tielliu IF, Slart RH, et al. Current state of experimental imaging modalities for risk assessment of abdominal aortic aneurysm. J Vasc Surg 2013; 57: $851-859$.

3. Nakayama A, Morita H, Hayashi N, Nomura Y, Hoshina K, Shigematsu K, et al. Inverse correlation between calcium accumulation and the expansion rate of abdominal aortic aneurysms. Circ J 2016; 80: 332-339.

4. Speelman L, Bohra A, Bosboom EM, Schurink GW, van de Vosse FN, Makaorun MS, et al. Effects of wall calcifications in patientspecific wall stress analyses of abdominal aortic aneurysms. J Biomech Eng 2007; 129: 105-109.

5. Buijs RV, Willems TP, Tio RA, Boersma HH, Tielliu IF, Slart RH, et al. Calcification as a risk factor for rupture of abdominal aortic aneurysm. Eur J Vasc Endovasc Surg 2013; 46: 542-548.

6. Sakalihasan N, Van Damme H, Gomez P, Rigo P, Lapiere CM, Nusgens B, et al. Positron emission tomography (PET) evaluation of abdominal aortic aneurysm (AAA). Eur J Vasc Endovasc Surg 2002; 23: $431-436$.

7. Kuivaniemi H, Ryer EJ, Elmore JR, Hinterseher I, Smelser DT, Tromp G. Update on abdominal aortic aneurysm research: From clinical to genetic studies. Scientifica (Cairo) 2014; 2014: 564734.

8. New SE, Aikawa E. Cardiovascular calcification: An inflammatory disease. Circ J 2011; 75: 1305-1313.

9. Fukuda S, Watanabe H, Iwakura K, Daimon M, Ito H, Yoshikawa J. Multicenter investigations of the prevalence of abdominal aortic aneurysm in elderly Japanese patients with hypertension. Circ J 2015; 79: $524-529$. 